\title{
Clinical Profiles of Thyroid Dermopathy: A Dermato-Endocrinological Perspective
}

\author{
Taoreed Adegoke Azeez', Ayobami Chioma Egbu² \\ 1 Endocrinology Unit, Department of Medicine, University College Hospital, Ibadan, Nigeria \\ 2 Dermatology Unit, Department of Medicine, University College Hospital, Ibadan, Nigeria
}

\section{CORRESPONDENCE}

\section{Taoreed Azeez}

Queen Elizabeth Road

Oritamefa, Ibadan, Oyo State, Nigeria

Tel: +23 47035728747

E-mail: adegokegalaxy@yahoo.com

\section{ARTICLE HISTORY}

Received: September 22, 2020

Accepted: October 26, 2020

\begin{abstract}
Thyroid disorders sometimes have extra-thyroidal manifestations. Hyperthyroidism is a clinical syndrome resulting from excessive secretion of thyroid hormones. The most common cause is Graves' disease. About 0.5-4.3\% of patients with Graves' disease have an infiltrative dermopathy called thyroid dermopathy, which is due to excessive deposition of glycosaminoglycans from activated fibroblasts. Skin fibroblasts are activated by thyroid stimulating hormone receptor antibodies the whole process being initiated by T lymphocytes. Rarely, thyroid dermopathy is also found in other thyroid disorders such as Hashimoto thyroiditis. The diffuse non-pitting edema variant is the most common clinical presentation. Other variants include the nodular, plaque, mixed, and elephantiasis types. Usually, the main concerns of the patients are cosmetic, discomfort, and difficulty in wearing shoes. Thyroid dermopathy usually presents after the diagnosis of Graves' disease, but it may also present together or sometime after this condition. Rarely, thyroid dermopathy presents before the diagnosis of Graves' disease is made. Apart from the shin and feet, other sites that can be affected include the arms, forearms, back, thighs, pinna, and nose. The management is multidisciplinary, involving dermatologists and endocrinologists. Usually, controlling the thyroid dysfunction does not translate into regression of the skin lesions. However, many patients have their thyroid dermopathy regress spontaneously, while others usually require local therapy. Other therapeutic options include systemic therapy such as pentoxifylline, compressive physiotherapy, and surgery.
\end{abstract}

Keywords: thyroid dermopathy, clinical profile, dermato-endocrinological perspective

\section{INTRODUCTION}

The thyroid gland is a butterfly-shaped endocrine organ located in the neck. It produces thyroid hormones, which regulate metabolic activities in the body. Hyperthyroidism is a clinical syndrome caused by excessive production and secretion of thyroid hormones. The commonly reported clinical features are heat intolerance, anxiety, excessive sweating, palpitation, fatigue, hyperdefecation, and menstrual disturbance. ${ }^{1}$ Graves' disease, an autoimmune disorder, is the most common cause of hyperthyroidism. ${ }^{2}$ Occasionally, it affects other organs such as the eyes and the skin. 
Thyroid dermopathy, sometimes called pretibial myxedema, is found in about $0.5-4.3 \%$ of patients with Graves' disease. ${ }^{3}$ It is an infiltrative dermopathy diagnosed in the presence of typical symptoms and signs, biochemical evidence of thyroid dysfunction or evidence of the characteristic autoimmune markers, as well as pathological findings in the skin. ${ }^{3}$ It manifests in various ways, affecting the skin of different parts of the body.

\section{PATHOGENESIS}

In genetically predisposed individuals, thyroid dermopathy results from an immunological cross-reaction occurring in the skin, where thyroid stimulating hormone (TSH) receptor antibodies stimulate the fibroblasts in the connective tissue. ${ }^{4}$ In vitro studies have demonstrated that fibroblasts in the skin and orbit express TSH receptors on their cell surface. ${ }^{5}$

Activation of the fibroblasts make them overproduce glycosaminoglycans, leading to fluid retention in the connective tissue of the skin. The antigen-antibody response is initiated by the activation of T lymphocytes. Other factors, such as localized trauma and venous stasis, have also been suggested to contribute to the development of thyroid dermopathy.

Histopathologically, reduced collagen fibers, extensive mucin deposition, reduced elastic tissue, and edema

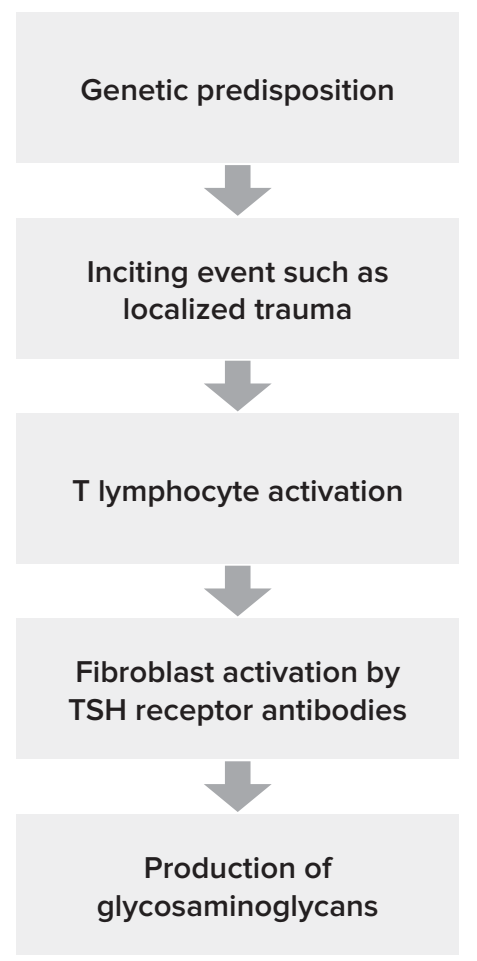

FIGURE 1. The pathogenesis of thyroid dermopathy are the characteristic findings. ${ }^{3}$ Other rarely documented pathological features include hyperkeratosis, acanthosis, and papillomatosis. ${ }^{3}$ The pathogenesis of thyroid dermopathy is summarized in Figure 1.

\section{RISK FACTORS}

Risk factors for the development of thyroid dermopathy are not known. However, some researchers have suggested that previous radioiodine therapy, smoking, thyroid hormonal imbalance, as well as the presence of Graves' orbitopathy could predispose an individual to developing thyroid dermopathy. ${ }^{7}$

\section{SITES OF THYROID DERMOPATHY}

The term 'pretibial myxoedema' is less favored to thyroid dermopathy because the skin lesions are found in many other areas apart from the pretibial area. The most common sites documented in the literature and their frequency are illustrated in Table $1 .^{3}$ The reasons for lower limb predilection are not known. Some hypotheses include gravitational forces and a higher concentration of fibroblasts. ${ }^{3}$

\section{CLINICAL PRESENTATION OF THYROID DERMOPATHY}

Thyroid dermopathy is found mostly in Graves' disease but it has also been documented in patients with Hashimoto thyroiditis, atrophic thyroiditis, and in euthyroid individuals. ${ }^{6}$ It is also more frequently seen in females compared to males. This may be due to the fact that autoimmune thyroid disorders are generally more common in females. ${ }^{3}$ The main reasons for presentation include cosmetic complaints, discomfort, and difficulty in wearing shoes.

TABLE 1. Sites of thyroid dermopathy and their frequencies

\begin{tabular}{ll}
\hline Site & Frequency \\
\hline Pretibial only & $94 \%$ \\
Pretibial + feet & $4 \%$ \\
Pretibial + upper extremities & $<1 \%$ \\
Arms & $<1 \%$ \\
Preradial forearms & $<1 \%$ \\
Upper back & $<1 \%$ \\
Shoulder & $<1 \%$ \\
Pinnae & $<0.5 \%$ \\
Nose & $<0.01 \%$ \\
Thigh & $<0.01 \%$ \\
\hline
\end{tabular}


The temporal profile of thyroid dermopathy in relation with Graves' disease is variable. Usually, it occurs after the diagnosis of Graves' disease but it may also predate it, or they may present concurrently. ${ }^{3}$ Often, it occurs after the onset of Graves' orbitopathy, another manifestation of Graves' disease with similar pathophysiology.

Thyroid dermopathy needs to be differentiated from lichen planus, stasis dermatitis, lichen simplex chronicus, and necrobiosis lipoidica. Thus, a review by a dermatologist is crucial in the management of thyroid dermopathy.

\section{TYPES OF THYROID DERMOPATHY}

Thyroid dermopathy presents in various forms. The various clinical variants are discussed briefly below. Figure 2 illustrates the clinical variants of thyroid dermopathy and their frequencies, based on the findings of a retrospective study conducted by Sabanova et al. ${ }^{8}$

The diffuse thyroid dermopathy variant usually presents as non-pitting edema. It is the most common variant and is characterized by induration of the skin giving the classical 'peau d' orange' sign. ${ }^{3}$ Some patients, in addition to the peau d'orange sign, also have diffuse hyperpigmented papules. Other variants are nodular and diffuse plaque types. Rarely, patients present with mixed nodular and plaque type. The elephantiasis variant, which presents like the late stage of lymphatic filariasis, has also been documented. ${ }^{8}$ This is often accompanied by significant functional disability. The complications of thyroid dermopathy include psychological distress, superimposed bacterial infection, recurrence, and entrapment neuropathy causing foot drop. ${ }^{3}$

\section{TREATMENT OF THYROID DERMOPATHY}

The treatment of thyroid dermopathy requires a collaborative effort from the endocrinologist and the dermatologist. The treatment is often challenging; in many patients, the duration of treatment requires months before regression begins, but they mostly regress eventually. ${ }^{4}$ Studies have shown that control of the thyroid dysfunction may not necessarily lead to the regression of thyroid dermopathy. ${ }^{2}$ Topical steroids, and in some instances intralesional steroid, with or without occlusion, have been used with some moderate response. In some severe variants, compressive physiotherapy as well as surgery have been adopted as therapeutic options. Newer therapies include the use of pentoxifylline and somatostatin analogues to reduce the production of glycosaminoglycans. However, there is a paucity of data on the outcomes of these treatment mo-

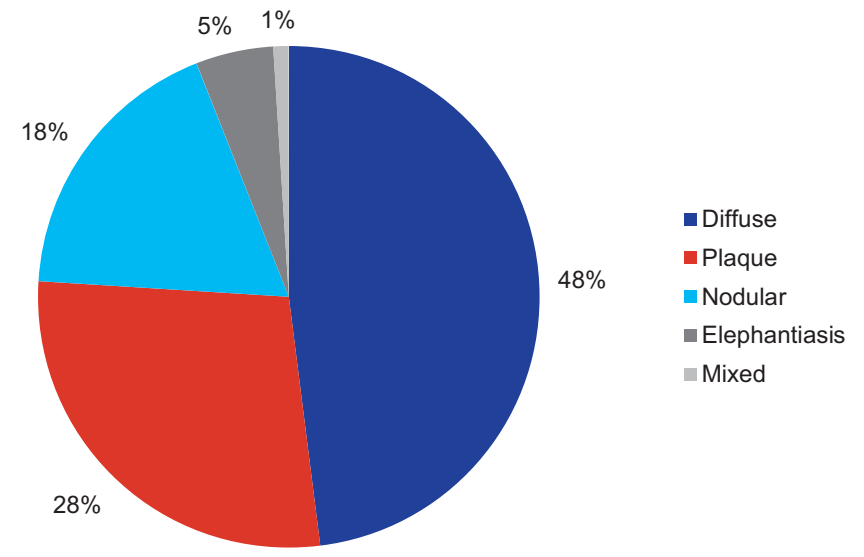

FIGURE 2. Thyroid dermopathy variants and their frequencies ${ }^{8}$

dalities. In resistant cases, intravenous globulins or plasmapheresis have been attempted. ${ }^{3}$

\section{PROGNOSIS}

Largely, the prognosis of thyroid dermopathy is good. A study done by Schwartz et al., assessing the long-term outcome of thyroid dermopathy, reported that close to half of the patients in the series regressed spontaneously, without requiring any intervention. ${ }^{4}$ The majority of patients requiring therapy had only topical steroids or intralesional steroids, and most of them had a significant response to therapy in form of partial or complete regression.

\section{CONCLUSIONS}

Thyroid dermopathy is an infiltrative dermopathy caused by the accumulation of glycosaminoglycans deposited by skin fibroblasts activated by TSH receptorstimulating antibodies. They are found in less than $5 \%$ of Graves' disease patients. The most frequent presentation is diffuse non-pitting edema. Other variants include nodular, plaque, elephantiasis, and mixed types. The most common sites involved are the pretibial area and feet, while other sites, such as the upper limbs, back, and thighs, are rarely involved. Thyroid dermopathy resolves spontaneously in many patients. Local therapy is the most commonly employed treatment modality, and management requires inputs from the dermatologist and the endocrinologist.

\section{CONFLICT OF INTEREST}

None declared. 


\section{REFERENCES}

1. Adeleye JO, Emuze ME, Azeez TA, Esan A, Akande TO, Balogun WO Clinical profiles of males with Graves' disease: a two year review in a tertiary hospital in Nigeria. J Clin Biomed Res. 2020;2:1-2.

2. Samuels $\mathrm{MH}$. Most patients with Graves' disease treated with additional anti-thyroid drugs eventually require additional therapies. Clin Thyroidol. 2020;32:9-11.

3. Dhali TK, Chahar M. Thyroid dermopathy - a diagnostic clue of hidden hyperthyroidism. Dermatoendocrinol. 2014;6:e981078.
4. Schwartz KM, Fatourechi V, Ahmed DD, Pond GR. Dermopathy of Graves' disease (pretibial myxedema): long-term outcome. J Clin Endocrinol Metab. 2002;87:438-446.

5. Davies T, Marians R, Latif R. The TSH receptor reveals itself. J Clin Invest 2002;110:161-164

6. Nair PA, Mishra A, Chaudhary A. A pretibial myxedema associated with euthyroid Hashimoto thyroiditis: a case report. J Clin Diagn Res. 2014;8:1-2.

7. Peacey SR, Flemming L, Messenger A, Weetman AP. Is Graves' dermopathy a generalized disorder? Thyroid. 1996;6:41-45.

8. Sabanova E, Fadeyev V, Lvov A. Thyroid dermopathy: clinical features in Graves' disease. Endocrine Abstracts. 2019;63:1217. 